\title{
Educação Interprofissional em Saúde: análise bibliométrica da produção científica nacional
}

\author{
Cibele Michel*; Thaís Ostroski Olsson**; Ramona Fernanda Ceriotti Toassi***
}

* Assistente Social da Prefeitura Municipal de Parobé, Rio Grande do Sul, especialista em Saúde Pública pela Universidade Federal do Rio Grande do Sul

** Bolsista de Iniciação Científica, estudante de graduação em Odontologia, Universidade Federal do Rio Grande do Sul

*** Doutora em Educação, Professora Associada do Departamento de Odontologia Preventiva e Social da Faculdade de Odontologia e do Programa de Pós-graduação em Ensino na Saúde, Universidade Federal do Rio Grande do Sul

Recebido em 11/10/2018. Aprovado em 23/12/2019.

\begin{abstract}
RESUMO
Educação interprofissional (EIP) é uma abordagem pedagógica importante nos currículos da saúde por buscar preparar os estudantes para o cuidado em um ambiente de equipe colaborativa. Este artigo propôs-se a realizar uma análise bibliométrica da produção científica em relação à EIP em saúde no Brasil, de 2012 a 2017. A pesquisa foi realizada na base de dados da Biblioteca Virtual em Saúde (BVS). Como descritor controlado foi utilizado o termo 'Relações interprofissionais/ Interprofessional Relations' e como descritores não controlados 'Educação Interprofissional/ Interprofessional Education', 'Aprendizado Colaborativo/ Collaborative Learning' e 'Trabalho em Equipe/ Teamwork'. Na busca, esses descritores foram combinados e acrescidos do operador boleano 'AND'. Foram analisadas 46 publicações (37 artigos, 6 dissertações, 3 teses). Os primeiros autores eram brasileiros, vinculados a Instituições de Ensino Superior público (78,2\%). Periódicos da Enfermagem, Saúde Pública e Educação, Qualis B, se destacaram entre os artigos analisados. Os estudos mais frequentes foram os de abordagem qualitativa (61\%), realizados na região Sudeste $(58,7 \%)$, cujos participantes foram profissionais da saúde $(23,9 \%)$ e estudantes de graduação, atualização, especialização e residência (19,5\%). A 'associação de técnicas' (24\%), seguida pela entrevista semiestruturada $(21,7 \%)$ foram as técnicas de coleta de dados mais observadas. Análise de conteúdo foi citada em $41,3 \%$ das publicações como a forma de análise dos dados. Esses estudos tratavam de experiências de EIP voltadas ao ensino na saúde (45,6\%), EIP nos serviços de Atenção Primária à Saúde $(23,9 \%)$, e EIP nos espaços de ambulatório e hospital $(15,1 \%)$. Seis publicações apresentaram fonte de financiamento. Pesquisas sobre EIP são incipientes no país, devendo ser ampliadas e qualificadas.
\end{abstract}

Descritores: Relações Interprofissionais. Educação Superior. Currículo. Bibliometria. 


\section{INTRODUÇÃO}

$\mathrm{O}$ aumento da complexidade das demandas em saúde convoca a uma nova compreensão do 'fazer saúde', na qual é necessário o rompimento de práticas fragmentadas nos serviços de saúde e a efetivação de novos modelos de educação, os quais, historicamente, estabeleceram-se de forma uniprofissional ${ }^{1}$.

No Brasil, como o cuidado integral está representado na essência da construção do Sistema Único de Saúde (SUS) e o 'fazer saúde' apresentase dinâmico e complexo, o debate sobre trabalho em equipe sempre esteve presente, ainda que, com diferentes enfoques no processo de formação dos profissionais da saúde ${ }^{2}$. Há, entretanto, um distanciamento entre as práticas de saúde e os modelos de educação na saúde. $\mathrm{O}$ processo educativo permanece sendo realizado, em sua grande maioria, apenas com formações específicas dentro de cada categoria profissional. São modelos desfavoráveis ao direcionamento das políticas públicas de saúde no Brasil, as quais exigem na prática dos serviços e nos processos de trabalho, ações cada vez mais colaborativas entre as diferentes profissões ${ }^{3}$.

Considerando esse contexto e buscando estratégias de ensino-aprendizagem que melhorem a qualidade da atenção à saúde a partir do trabalho colaborativo entre diferentes profissionais, a educação interprofissional (EIP) passa a ser um tema emergente, sendo considerada uma abordagem pedagógica importante nos currículos da saúde ${ }^{4-6}$.

A EIP ocorre quando integrantes de mais de uma profissão da saúde aprendem em conjunto, de forma interativa, com o propósito explícito de melhorar a colaboração interprofissional ou a saúde/bem-estar das pessoas ${ }^{7}$.

Esta pesquisa teve o objetivo de realizar uma análise bibliométrica da produção científica nacional em relação à EIP em saúde, no período de 2012 a 2017.

\section{METODOLOGIA}

Trata-se de um estudo bibliométrico sobre o tema da 'Educação interprofissional em saúde no Brasil' que utilizou a base de dados bibliográficos da Biblioteca Virtual em Saúde - BVS (<http://www.bireme.br>), a qual inclui as seguintes fontes de informação: Literatura Latino-Americana e do Caribe em Ciências da Saúde (LILACS), Índice Bibliográfico Espanhol de Ciências da Saúde (IBECS), Medical Literature Analysis and Retrieval System Online (MEDLINE) e Scientific Electronic Library Online (SciELO).

Estudos bibliométricos buscam colaborar com a investigação e avaliação da produção científica, mensurando a produtividade do campo acadêmico, apontando padrões das publicações, principais áreas de desenvolvimento e reconhecimento de investigadores e autorias ${ }^{8}$.

A identificação dos descritores controlados para a busca foi realizada junto ao vocabulário estruturado Descritores em Ciências da Saúde (DeCS). Foram utilizados como descritores controlados os termos 'Relações interprofissionais/ Interprofessional Relations' e como descritores não controlados 'Educação Interprofissional/ Interprofessional Education', Aprendizado Colaborativo/ Collaborative Learning' e Trabalho em Equipe/ Teamwork'. Esses descritores foram combinados e acrescidos do operador boleano 'AND' para a realização da busca, resultando em quatro combinações, conforme o quadro 1 .

No campo de pesquisa os descritores deveriam constar no título, resumo ou assunto das publicações. O período de tempo definido para a busca foi de 2012 a 2017. A Organização Mundial da Saúde publicou importante documento que constituiu um marco de referência para ampliar o debate sobre EIP em nível mundial, o qual foi traduzido para o português em $2010^{1}$, dando destaque ao tema no país. Assim, optou-se por iniciar essa pesquisa em 2012, passados dois anos da publicação em português desse documento orientador.

Como critérios de inclusão, as publicações deveriam ser artigos, trabalhos acadêmicos ou ensaios, em português, inglês ou espanhol, 
envolvendo a temática estudada 'EIP no Brasil'. não abordavam a temática no Brasil. Após as Foram encontrados 176 artigos, 22 teses e 6 exclusões, 37 artigos, 3 teses e 6 dissertações foram dissertações. Destes trabalhos, 41 encontravam-se selecionadas, os quais foram obtidos na íntegra para duplicados entre as bases pesquisadas, 117 artigos análise (figura 1).

Quadro 1. Combinações utilizadas na estratégia de busca

\begin{tabular}{|r|} 
Combinação 1: Relações Interprofissionais AND Educação Interprofissional \\
Interprofessional Relations AND Interprofessional Education \\
Combinação 2: Relações Interprofissionais AND Aprendizado Colaborativo \\
Interprofessional Relations AND Collaborative Learning \\
Combinação 3: Relações Interprofissionais AND Trabalho em Equipe \\
Interprofessional Relations AND Teamwork \\
Combinação 4: Educação Interprofissional AND Aprendizado Colaborativo \\
Interprofessional Education AND Collaborative Learning \\
Combinação 5: Educação Interprofissional AND Trabalho em Equipe \\
Interprofessional Education AND Teamwork \\
Combinação 6: Aprendizado Colaborativo AND Trabalho em Equipe \\
Collaborative Learning AND Teamwork
\end{tabular}

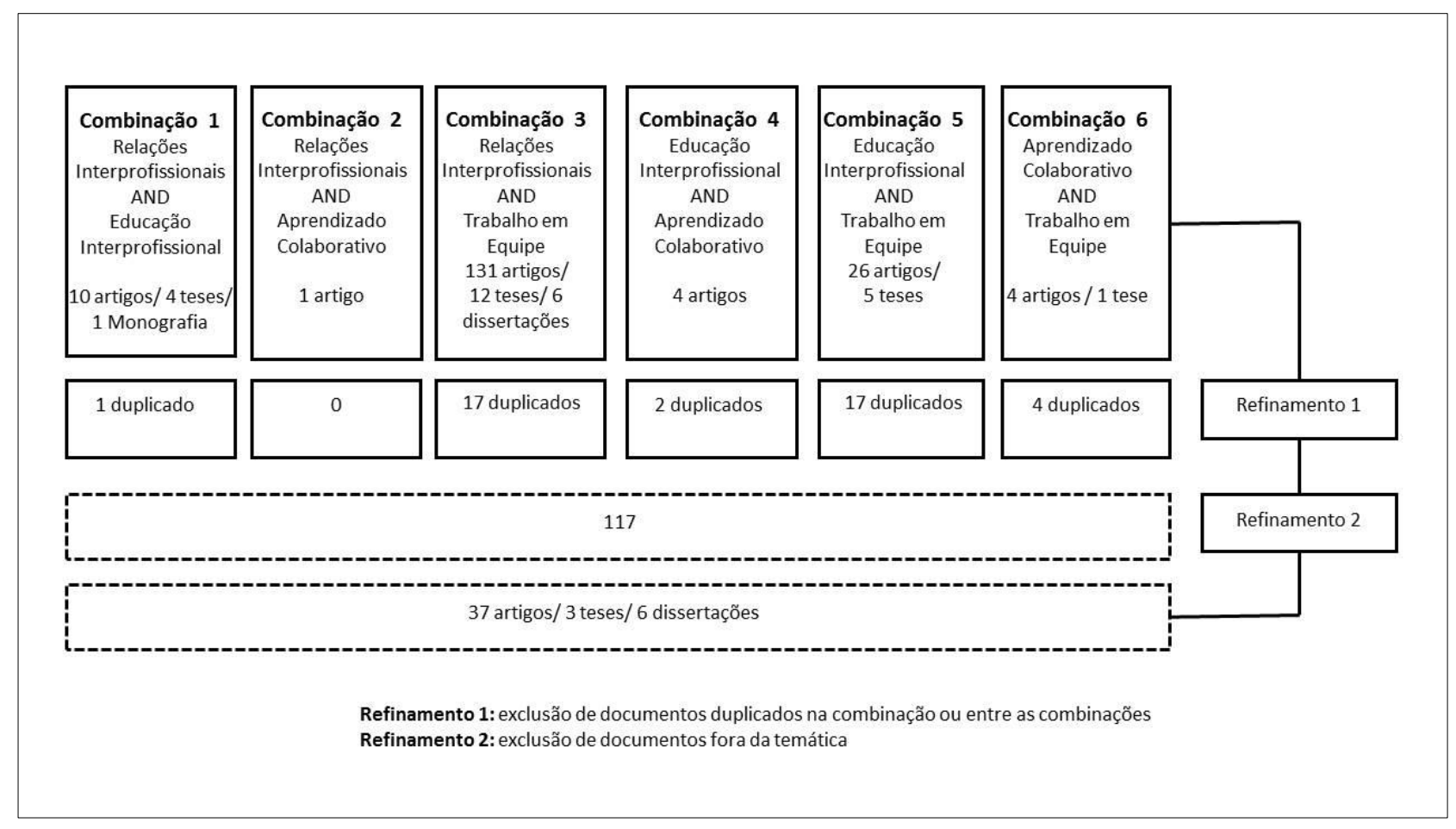

Figura 1. Sistematização do processo de seleção das publicações

As publicações selecionadas foram lidas na íntegra e analisadas de acordo com as seguintes variáveis:

- indicadores de atividade científica, os quais permitem contabilizar a atividade científica desenvolvida $^{8}$ (número de publicações e impacto das revistas e o Qualis Capes); distribuição por ano, número de autores por artigo e colaboração na autoria de trabalhos, vínculo de trabalho do $1^{\circ}$ autor, produtividade dos autores); - indicadores de impacto científico das fontes ${ }^{8}$ (periódico de publicação; área do periódico; fator de - DOI: $10.30979 /$ rev.abeno.v19i4.781 
- perfil das publicações (tipologia, local de realização dos estudos, participantes de pesquisa, técnica de coleta e análise de dados, fonte de financiamento e recortes temáticos).

Foi utilizado o programa Microsoft Excel versão 2010 para a construção de planilha eletrônica com a síntese das publicações e cálculo das frequências.

A pesquisa foi aprovada pela Comissão de Pesquisa (COMPESQ) da Faculdade de Odontologia da UFRGS (projeto 32428).

\section{RESULTADOS}

Foram analisadas 46 publicações, sendo 37 artigos, 6 dissertações e 3 teses provenientes da base BVS, voltados à EIP no Brasil, no período de 2012 a 2017. O resultado dessa análise está apresentado de acordo com os indicadores de atividade científica, indicadores de impacto científico das fontes e perfil das publicações.

\section{Indicadores de atividade científica}

Em relação ao número de publicações por ano, observou-se pouca variação de 2012 a 2015 (de 6 a 8). Houve, entretanto, aumento no número de publicações sobre a EIP em $2016(n=15)$ e redução em 2017 (n=3). O número de autores por publicação variou de um a sete (média de quatro autores por publicação), totalizando 157 autores para as 46 publicações. A maior parte das publicações analisadas apresentou entre um e três autores $(54,3 \%)$, sendo que $74 \%$ desses autores tiveram uma única publicação no período estudado (tabela 1).

Os primeiros autores das publicações eram brasileiros e, na sua maioria $(78,2 \%)$, estavam vinculados a diferentes Instituições de Ensino Superior (IES) público do país. Apenas em um artigo não foi observado vínculo do primeiro autor com IES e sim com serviço público de saúde (tabela 1).

Tabela 1. Distribuição das publicações sobre EIP no Brasil segundo indicadores de atividade científica, 2012-2017

\begin{tabular}{lcc}
\hline Variáveis & $\mathbf{n}$ & $\mathbf{\%}$ \\
\hline Ano de publicação & 6 & 13,0 \\
2012 & 8 & 17,4 \\
2013 & 7 & 15,2 \\
2014 & 7 & 15,2 \\
2015 & 15 & 32,6 \\
2016 & 3 & 6,5 \\
2017 & & \\
Número de autores & 25 & 54,3 \\
1 a 3 & 20 & 43,5 \\
4 a 6 & 1 & 2,2 \\
7 & & \\
Produtividade dos autores & 34 & 74,0 \\
Autoria em uma publicação & 8 & 17,3 \\
Autoria em duas publicações & 3 & 6,5 \\
Autoria em três publicações & 7 & 15,2 \\
Autoria em quatro publicações & & \\
Vínculo do primeiro autor & 36 & 78,2 \\
Instituição de Ensino Superior pública & 7 & 15,2 \\
Instituição de Ensino Superior privada & 2 & 4,4 \\
Instituição de Ensino Superior pública e serviço público de saúde & 1 & 2,2 \\
Serviço público de saúde & $\mathbf{4 6}$ & $\mathbf{1 0 0 , 0}$ \\
\hline Total & & \\
\hline
\end{tabular}


Mais da metade das IES dos autores dos trabalhos analisados estavam localizadas na região Sudeste do país $(56,7 \%)$. A Universidade de São Paulo apareceu vinculada a $23,9 \%$ das publicações avaliadas, a Universidade Federal de Minas Gerais e a Universidade Federal de São Paulo a $8,7 \%$ publicações cada.

Ainda em relação aos autores, foi possível identificar parcerias interinstitucionais em 30,4\% das publicações sobre EIP. Estiveram presentes também parcerias entre autores vinculados a instituições internacionais no Canadá, Inglaterra e Costa Rica e parcerias entre autores vinculados a instituições de ensino e serviços de saúde.

Entre aos autores com maior número de publicações no período, destacam-se Nildo Alves Batista $^{9-12}$ e Sylvia Helena Souza da Silva Batista $^{9,11,13,14}$ com quatro artigos publicados, Ana Ecilda Lima Ellery com uma tese de doutorado $^{15}$ e dois $\operatorname{artigos}^{16,17}$, Ana Claudia Camargo Gonçalves Germani com três $\operatorname{artigos}^{5,18,19}$, Marina Peduzzi com três $\operatorname{artigos}^{3,5,20}$, Jaqueline Alcântara Marcelino da Silva $^{5,20}$, Geisa Colebrusco de Souza ${ }^{5,20}$, Alfredo Almeida Pina de Oliveira ${ }^{18,19}$, Simone Coelho Amestoy $^{21,22}$, Michelle Barboza Jacondino ${ }^{21,22}$, Ana Maria Chagas Sette Camara ${ }^{14,23}$ e Franklin Delano Soares Forte ${ }^{24,25}$, com dois artigos.

\section{Indicadores de impacto científico das fontes}

Entre os 37 artigos analisados sobre a temática da EIP no Brasil, esses foram publicados especialmente em periódicos da área de Enfermagem $(\mathrm{n}=12$ - 21,1\%), Saúde Pública/Saúde Coletiva $(\mathrm{n}=12-21,1 \%)$ e Educação $(n=9-19,6 \%)$. Tais periódicos estavam, em sua maioria, classificados como Qualis B para a área da Saúde Coletiva, sendo 23,9\% B1, 21,7\% B2 e 21,7\% B4. Não foram identificados artigos publicados em periódicos Qualis A para essa mesma área de avaliação. Os periódicos com maior Fator de Impacto foram os da área da Enfermagem: Revista Brasileira de Enfermagem da USP, Revista Brasileira de
Enfermagem e Revista Gaúcha de Enfermagem (tabela 2).

\section{Perfil das publicações}

Os estudos de abordagem qualitativa foram os mais referidos nas publicações sobre a EIP no Brasil (61,0\%), seguido pelos relatos de experiência $(13,0 \%)$ e estudos quantitativos $(13,0 \%)$. Esses estudos foram realizados, em sua maioria, na região Sudeste do país $(58,7 \%)$, tendo como participantes profissionais da saúde (23,9\%), estudantes de graduação, atualização, especialização e residência $(19,5 \%)$ e profissionais da saúde e estudantes (15,2\%). Esses estudos apresentaram grande variabilidade quanto às técnicas de coleta de dados, sendo a associação de técnicas, a forma mais observada $(24,0 \%)$, seguida pela entrevista semiestruturada (21,7\%). A análise de conteúdo foi citada como a técnica de análise de dados em 41,3\% das publicações. Seis das 39 publicações mencionaram fonte de financiamento (tabela 3 ).

A diversidade de temáticas apresentadas nas publicações analisadas pode ser observada na tabela 4. Verificou-se um número maior de trabalhos sobre EIP voltados ao ensino na saúde, envolvendo graduação, pós-graduação e residência multiprofissional em saúde $(45,6 \%)$, seguido pela EIP nos Serviços de Atenção Primária à Saúde (APS), envolvendo toda equipe da Estratégia Saúde da Família (ESF) e os agentes comunitários de saúde (ACS), técnicos em saúde bucal, dentistas, e os profissionais do Núcleo de Apoio à Saúde da Família - NASF $(23,9 \%)$ e, em número menor, a EIP nos espaços de ambulatório e ambiente hospitalar (15,1\%). $\mathrm{Na}$ temática do ensino, enfatizam-se experiências de formação com foco interprofissional. Três artigos tratam de experiências no Programa de Educação para o Trabalho na Saúde (PETSaúde). Já nas publicações envolvendo os serviços de saúde, destaca-se o processo de trabalho em equipe multiprofissional e relações interprofissionais como temas recorrentes. 
Tabela 2. Distribuição das publicações sobre EIP no Brasil segundo indicadores de impacto científico das fontes (periódicos), 2012-2017 ( $\mathrm{n}=46)$

\begin{tabular}{lccc}
\hline Variáveis & \multicolumn{2}{c}{ Frequência } & \multirow{2}{*}{ FI* $^{*}$} \\
\hline Periódico de publicação/Assunto & $\mathrm{n}$ & $\mathbf{\%}$ & \\
Interface: comunicação, saúde e educação/Educação, Saúde Pública & 5 & 10,9 & 0,3056 \\
Revista Escola de Enfermagem da USP/Enfermagem & 4 & 8,7 & 0,3277 \\
ABCS Health Sciences/Medicina, Saúde Pública & 3 & 6,5 & -- \\
Revista de pesquisa: cuidado é fundamental (Online)/Enfermagem & 3 & 6,5 & -- \\
Revista Brasileira de Educação Médica/Educação & 2 & 4,3 & 0,0755 \\
Revista Brasileira de Enfermagem/Enfermagem & 2 & 4,3 & 0,5181 \\
Revista CEFAC/Patologia da Fala e Linguagem & 2 & 4,3 & 0,1624 \\
Revista de Medicina (São Paulo)/Medicina & 2 & 4,3 & \\
Revista Gaúcha de Enfermagem/Enfermagem & 2 & 4,3 & 0,3146 \\
Einstein (São Paulo)/Medicina & 1 & 2,2 & 0,0405 \\
Jornal Brasileiro de Telesaúde/Medicina & 1 & 2,2 & -- \\
Physis/Saúde Pública & 1 & 2,2 & 0,0667 \\
Psicologia em Revista/Psicologia & 1 & 2,2 & -- \\
Psicologia, ciência e profissão/Psicologia & 1 & 2,2 & 0,0938 \\
Revista Brasileira de Cancerologia/Neoplasias & 1 & 2,2 & -- \\
Revista da ABENO/Odontologia & 1 & 2,2 & -- \\
Revista da Rede de Enfermagem do Nordeste/Enfermagem & 1 & 2,2 & -- \\
Revista de Odontologia da UNESP/Odontologia & 1 & 2,2 & 0,0250 \\
Saúde em Debate/Saúde Coletiva & 1 & 2,2 & 0,2124 \\
Tempus Actas de Saúde Coletiva/Educação & 1 & 2,2 & -- \\
Trabalho, educação e saúde/Saúde Pública, Saúde Ambiental, Medicina, & 1 & 2,2 & 0,3038 \\
Não se aplica (Dissertações e Teses) & 9 & 19,6 & -- \\
Classificação Qualis Capes** & & & \\
A & -- & -- & \\
B1 & 11 & 23,9 & -- \\
B2 & 10 & 21,7 & -- \\
B3 & 3 & 6,5 & -- \\
B4 & 10 & 21,7 & -- \\
B5 & 2 & 4,3 & -- \\
C Não se aplica (Dissertações e Teses) & 1 & 2,2 & -- \\
Nand & 9 & 19,6 & -- \\
\hline
\end{tabular}

*FI = Fator de Impacto. É calculado pela divisão do número de vezes em que os artigos de uma dada revista indexada são citados em um determinado ano pelo número de trabalhos publicados por essa revista nos dois anos anteriores. Dados consultados dos Relatórios de citações de revistas, Fator de impacto em um período de dois anos, ano base 2018.

**Qualis Capes consultado para a área da Saúde Coletiva.

Tabela 3. Perfil das publicações sobre EIP no Brasil quanto à tipologia, local de realização dos estudos, técnica de coleta e análise dos dados, fonte de financiamento, 2012-2017 ( $\mathrm{n}=46)$

\begin{tabular}{lrr}
\hline Variáveis & $\mathbf{n}$ & \multicolumn{1}{c}{$\%$} \\
\hline Tipologia dos estudos & & \\
Estudo qualitativo & 28 & 61,0 \\
Relato de experiência & 6 & 13,0 \\
Estudo quantitativo & 6 & 13,0 \\
Revisão de literatura & 3 & 6,6 \\
Estudo quali-quantitativo & 2 & 4,4 \\
Ensaio & 1 & 2,2 \\
\hline & & Continua
\end{tabular}


Local de realização dos estudos

Região Sudeste

Região Nordeste

Continuação

Região Sul

$27 \quad 58,7$

Região Norte

$7 \quad 15,2$

Região Centro-o

$6 \quad 13,0$

Não se aplica (Revisão de literatura e Ensaio)

Participantes dos estudos

Profissionais da saúde

Estudantes (graduação, atualização, especialização e residência)

Profissionais da saúde e estudantes

Profissionais da enfermagem

Usuários do SUS

Gestores, profissionais da saúde e das IES

Estudantes, docentes (tutores) e preceptores

Estudantes e docentes

Técnicos em Saúde Bucal

Cirurgiões-dentistas

Não se aplica (Revisão de literatura e Ensaio)

Técnica de coleta de dados

Entrevista semiestruturada

Questionário

Análise documental

Entrevista semiestruturada e observação

Busca em bases de dados (Revisão de Literatura)

Grupo focal

Entrevista aberta

Entrevista aberta, observação

Entrevista aberta, oficina

Entrevista aberta, diário de campo, análise documental

Entrevista semiestruturada, questionário online

Entrevista semiestruturada, observação, dinâmica de grupo

Entrevista semiestruturada, questionário, técnica do Gibi

Diário de campo, grupo tutorial

Escala RIPLS, grupo focal

Utilização de dados secundários

Readiness Interprofissional Learning Scale (RIPLS)

Objective Structured Clinical Examination (OSCE) e grupo focal

Não se aplica (Relatos de experiência e Ensaio)

Técnica de análise dos dados

Análise de conteúdo

Análise estatística

Análise de discurso

Abordagem de análise de textos em Ricoeur (1986)

Análise temática e estatística

Leitura crítica/em profundidade

Análise de conteúdo e Técnica do Incidente Crítico

Análise descritiva

Análise dos diários de campo e das contribuições durante os

Teoria Fundamentada dos Dados 
Tabela 4. Recortes temáticos das publicações sobre EIP no Brasil, 2012-2017 (n=46)

\begin{tabular}{lrrr}
\hline Recortes temáticos das publicações & n & \% \\
\hline EIP no ensino (graduação, residência e pós-graduação) & 21 & 45,6 \\
EIP nos Serviços de Atenção Primária à Saúde & 11 & 23,9 \\
EIP em ambulatório e ambiente hospitalar & 7 & 15,1 \\
EIP relacionadas ao SAMU* & 2 & 4,4 \\
Trabalho interdisciplinar na área da saúde & 1 & 2,2 \\
Análise dos construtos teóricos da EIP & & 1 & 2,2 \\
Relação entre paralisia facial e qualidade de vida, no âmbito do trabalho & 1 & 2,2 \\
interprofissional & & 1 & 2,2 \\
Percepção de profissionais de saúde em relação à colaboração interprofissional & 1 & 2,2 \\
Promoção da saúde em ambiente de trabalho
\end{tabular}

*SAMU: Atendimento Móvel de Urgência.

\section{DISCUSSÃO}

Esta pesquisa propôs-se a analisar a produção do conhecimento científico sobre a temática da EIP em saúde no Brasil, por meio de uma bibliometria.

A discussão sobre a EIP emerge no Brasil a partir do reconhecimento da complexidade das demandas em saúde e da necessidade do cuidado efetivo às pessoas. A compreensão das múltiplas dimensões presentes no processo saúde-doença, e não apenas a ausência da doença, exigiu a reorganização dos serviços e a colaboração dos profissionais em resposta a essa demanda. Surge, então, como um modelo de intervenção com potencial para melhorar a qualidade da atenção à saúde, por meio do efetivo trabalho entre diferentes profissionais, na perspectiva da prática colaborativa. A EIP é considerada uma estratégia essencial para formação de profissionais por estar em consonância com as diretrizes do SUS e possibilitar a realização do trabalho em equipe de forma mais eficaz ${ }^{2-4}$.

Para que a EIP ocorra, é preciso que estudantes ou profissionais de dois cursos ou núcleos profissionais aprendam sobre os outros (a respeito do fazer de outras categorias profissionais e seus objetos de trabalho), com os outros (de forma interativa contando com a coparticipação) e entre si (para a efetiva prática colaborativa e melhora dos resultados na saúde) ${ }^{1}$.

No presente estudo, 46 publicações sobre EIP no Brasil foram identificadas na base de dados da BVS, de 2012 a 2017, e analisadas. Observou-se um número discreto de publicações no período, chamando a atenção o ano de 2016, o qual teve um número maior de publicações sobre EIP, comparado com os demais anos $(n=15)$. Esse aumento se justifica pois, em 2016, a revista Interface publicou número com editorial que marca uma iniciativa de colaboração dos editores do Journal of Interprofessional Care e da Interface: Comunicação, Saúde, Educação para publicar editoriais em inglês e português sobre questões críticas relacionadas com o avanço da colaboração interprofissional no Brasil. O editorial destacou o I Colóquio Internacional de Educação e Trabalho Interprofissional em Saúde (CIETIS), realizado em Natal, Rio Grande do Norte, em julho de $2015^{26}$ e esse mesmo número da Interface ainda apresentou uma série de artigos sobre a EIP com pesquisadores de referência sobre a temática no Brasil e no contexto internacional ${ }^{2,3,27-}$ 29.

Embora haja no Brasil iniciativas de aprendizagem conjunta e políticas que colaboram para as mudanças no processo de formação na graduação, o achado desta bibliometria indica que, para o contexto brasileiro, o debate e as práticas apoiadas na EIP na área da saúde ainda são recentes, refletindo-se em uma baixa expressividade de publicações $^{4,30}$.

O perfil das publicações analisadas é marcado por primeiros autores brasileiros, vinculados especialmente a universidades públicas, localizadas na região Sudeste do país, reforçando a heterogeneidade espacial das atividades de pesquisa científica, onde o padrão regional da distribuição das 
publicações e dos pesquisadores é altamente concentrado na região Sudeste ${ }^{31}$.

As parcerias interinstitucionais identificadas no campo autoral em 30,4\% das 46 publicações sobre EIP, expressam a dificuldade de produções colaborativas entre as diferentes instituições.

Em relação aos artigos sobre EIP no Brasil analisados nesta pesquisa, observou-se que esses foram publicados em 21 periódicos distintos, sendo a 'Interface: comunicação, saúde e educação' e a 'Revista Escola de Enfermagem da USP' os periódicos de preferência para tais publicações. Desses periódicos, todos tinham classificação Qualis, no portal WebQualis, mas nenhum era Qualis A para a área da Saúde Coletiva. A fonte de financiamento foi apresentada em 6 das 46 publicações (13\%), mostrando um baixo investimento de pesquisas neste tema.

É oportuno destacar que as barreiras até então impostas a pesquisas envolvendo a interação de áreas de conhecimento pelo sistema de avaliação Qualis (base das avaliações de produtividade no Brasil), na qual havia critérios diferenciados de classificação da produção científica para cada campo disciplinar ${ }^{32}$, pode ter sido um fator que fragilizou parcerias interprofissionais e até estudos sobre este tema, fortalecendo pesquisas com caráter uniprofissional e disciplinar. A nova proposta para o Qualis Periódicos anunciada pela CAPES em julho de 2019, prevendo mudanças para uma classificação única - cada periódico recebe apenas uma qualificação, independentemente da quantidade de áreas de avaliação - tende a estimular o desenvolvimento de estudos com pesquisadores de distintos núcleos profissionais e campos disciplinares.

Outro aspecto que merece destaque nesta análise foi a preferência de opção pelas pesquisas de abordagem qualitativa, as quais foram as mais frequentes nas publicações. Cada vez mais difundida no campo da saúde, essas pesquisas têm como termos estruturantes um conjunto de substantivos cujos sentidos são complementares experiência, vivência, senso comum e ação - e os verbos compreender e interpretar. Ou seja, são pesquisas utilizadas quando o objeto de pesquisa exige respostas que não podem ser traduzidas em números, e sim na experiência, na vivência, no senso comum e na ação. Levam em conta a singularidade do indivíduo, por meio de sua subjetividade ${ }^{33}$, isto é, examinam a compreensão das pessoas a respeito do que experienciam na vida cotidiana ${ }^{34}$.

As temáticas das publicações analisadas focaram-se em trabalhos sobre EIP voltados, preferencialmente, ao ensino na saúde, ao espaço do trabalho em equipe nos serviços de APS/ ESF e de ambulatório, bem como ambiente hospitalar. Não houve, nas publicações analisadas, evidências que indicassem o melhor método para operacionalizá-la na prática. Em um dos estudos realizados, os resultados contemplaram o momento da formação para ser iniciada a EIP, mostrando que estudantes ingressantes apresentaram alta disponibilidade para a EIP, tornando o início da vida acadêmica favorável a essas atividades ${ }^{23}$.

$\mathrm{Na}$ temática do ensino, predominaram experiências positivas de formação com foco na EIP, incluindo experiências no Programa de Educação para o Trabalho na Saúde (PET-Saúde), o qual se configura como espaço privilegiado de integração entre as atividades tanto de atenção quanto da formação em saúde ${ }^{14,26}$. A maior crítica desses estudos, contudo, esteve voltada a mudanças nos currículos, os quais ainda se mantêm em muitas instituições com formato uniprofissional.

Estudo de Frenk et al. ${ }^{35}$ sobre a educação dos profissionais da saúde no século 21 , mostrou que, de modo geral, os currículos não se desenvolveram no ritmo dos desafios, apresentam-se ultrapassados, estáticos, fragmentados e conservadores, consequentemente resultam em profissionais não resolutivos, não melhorando o desempenho dos sistemas de saúde.

Pesquisas que problematizem os processos e modelos de formação são fundamentais para que ocorra a prática interprofissional, o que foi observado nessa pesquisa. A EIP convoca o pensar a respeito das mudanças dos currículos e metodologias de ensino, e consiste na inversão da lógica tradicional da formação em saúde - cada 
prática profissional pensada e discutida em si -, abrindo espaços para a discussão do interprofissionalismo ${ }^{36}$.

Revisão sistemática sobre EIP conduzida por Reeves et al. ${ }^{7}$ também mostrou que a mesma se faz cada vez mais presente nos serviços de saúde e nas instituições de ensino. Ela abrange diferentes níveis de formação, graduação, pós-graduação, educação continuada, cursos de extensão e esses modelos de aprendizagem têm ocorrido por meio de diferentes metodologias que contam com aulas presencias em salas de aula, ensino a distância, laboratórios virtuais e ambientes online. Nesse estudo, as duas publicações que trataram de tecnologias de informação e comunicação na área da saúde demonstraram novos modelos de ensino, facilidades e resistências ao uso desses dispositivos. Não apresentaram, entretanto, nesse momento, avaliação consistente sobre o impacto dessas tecnologias para a EIP.

Entende-se que o fortalecimento de pesquisas que apresentem e avaliem experiências interprofissionais, tanto no ensino quanto nos serviços de saúde, contribuem para consolidar 'o capital científico, ${ }^{, 37}$ sobre o tema, podendo subsidiar tanto mudanças no modelo de formação uniprofissional ainda vigente na saúde, quanto a formulação de políticas públicas de saúde ${ }^{37,38}$.

Resistências são enfrentadas para o rompimento desse modelo de formação pautado na forte divisão do trabalho e em categorias tradicionais da saúde. Os profissionais ainda continuam sendo formados em seus núcleos separadamente, para posteriormente trabalharem juntos, lógica contraditória que traz importantes implicações para qualidade da atenção oferecida no âmbito da saúde pública $^{2}$. É necessário que os estudantes de graduação se envolvam com essa estratégia pedagógica da EIP, ou seja, por esse novo formato que requer a contribuição de diferentes áreas e saberes profissionais. Sendo assim, pode haver múltiplos itinerários de aprendizagem, compreendendo os campos da observação, ação, troca, simulação e prática em contextos reais. A efetivação dos processos de EIP requer uma nova organização curricular, um redesenho do ensino, para este que seja adequado e compreenda a relevância das discussões e vivências coletivas por parte dos diferentes profissionais, as oportunidades de aprendizagens mútuas e as soluções coletivas, bem como os impactos desses processos na prática do cuidado em saúde. Isso inclui fluxos de conhecimento, tecnologias e financiamentos de pesquisas na área ${ }^{35,39}$.

Nesta pesquisa, as publicações que versaram sobre a prática dos trabalhadores no cotidiano dos serviços, abordaram diferentes categorias profissionais nos serviços de saúde, com foco no trabalho colaborativo, trabalho em equipe, cooperação interprofissional e relações interprofissionais. Novamente ficou evidenciado que os modelos de formação influenciam diretamente na prática podendo contribuir para os fazeres coletivos e as relações interprofissionais ou até mesmo reforçar a prática individualizada e hierarquizada dentro das equipes. Os resultados desses estudos indicavam que uma parcela significativa dos profissionais desconhecia $\mathrm{o}$ trabalho do outro e, consequentemente, a importância das demais categorias presentes no cuidado em saúde. Tampouco tinham vivenciado ações que se dão em equipe durante a graduação, o que não possibilitou uma avaliação dos possíveis efeitos das intervenções colaborativas entre as profissões da saúde, tema em ainda desenvolvimento e que precisa de estudos mais robustos para gerar evidências suficientes sobre essas intervenções interprofissionais ${ }^{40}$. A Enfermagem demonstrou maior proximidade ao trabalho colaborativo e até mesmo ao compartilhamento das responsabilidades.

Os achados dessa análise bibliométrica também evidenciaram que serviços de saúde específicos - como urgência, emergência, Estratégia de Saúde da Família -, apresentam melhores condições de desenvolver um trabalho colaborativo do que outros. Os estudos trazem os desafios da ausência de espaços para discussão de casos, inexistência de tempo para pensar as situações vistas às urgências expostas, a não compreensão da 
necessidade do trabalho interprofissional e o desconhecimento da potência existente nessa metodologia de trabalho compartilhado. Nas experiências interprofissionais onde o apoio organizacional está ausente ou limitado, normalmente ocorrem problemas para o desenvolvimento da EIP, o que inclui a falta de recursos financeiros, tempo, espaço instituído, espaço físico, a visão da organização e a missão pela qual ela se ocupa ${ }^{41}$.

\section{CONSIDERAÇÕES FINAIS}

Considerando a relevância da EIP para a reorganização das práticas colaborativas de cuidado em saúde e qualificação do trabalho em equipe, o discreto número de publicações encontrado sobre a temática no período estudado, a maioria publicada em periódicos nacionais (Qualis B), sem financiamento, indica a incipiência dessas pesquisas no Brasil.

Como esta análise bibliométrica optou por consultar exclusivamente a base de dados da BVS, considera-se esta, uma limitação do presente estudo. Recomenda-se, assim, ampliação dessa estratégia de busca envolvendo mais bases de dados, bem como a avaliação das ações desenvolvidas a partir da EIP no Brasil nas Instituições de Ensino Superior e no Sistema Único de Saúde.

\section{ABSTRACT \\ Interprofessional Health Education: bibliometric analysis of national scientific production}

Interprofessional education is an important pedagogical approach in health curricula. It aims at preparing students for care in a collaborative team environment. This paper is intended to conduct a bibliometric analysis of the scientific production in interprofessional health education in Brazil from 2012 to 2017. The research was conducted based on Virtual Health Library (BVS) data. The term relações interprofissionais (interprofessional relations) was used as controlled descriptor, and the terms educação interprofissional (interprofessional education), aprendizado colaborativo (collaborative learning), and trabalho em equipe (teamwork) were used as non-controlled descriptors. In the search, these descriptors were combined with the Boolean operator AND. A total of 46 publications (37 papers, 6 theses, 3 dissertations) were analyzed. The first authors were Brazilian and associated with public higher education institutions (78.2\%). Nursing, Public Health, and Education periodicals (Qualis B) were highlighted among the analyzed papers. The most frequent studies were of qualitative approach (61\%), conducted in the Southeastern region (58.7\%), with participants who were health professionals $(23.9 \%)$ and undergraduate, refresher, specialization, and residency students (19.5\%). "Association of techniques" (24\%) and semistructured interviews $(21.7 \%)$ were the most observed data collection techniques. Content analysis was mentioned in $41.3 \%$ of publications as a data analysis type. These studies were about interprofessional education focused on health education $(45.6 \%)$, interprofessional education in primary care services $(23.9 \%)$ and in outpatient clinics and hospitals (15.1\%). Six publications were funded. Interprofessional education research is incipient in Brazil and should be expanded and qualified.

Descriptors: Interprofessional relations. Education, Higher. Curriculum. Bibliometry.

\section{REFERÊNCIAS}

1. Organização Mundial da Saúde. Marco para Ação em Educação Interprofissional e Prática Colaborativa. 2010. 64 p.

2. Costa MV. A educação interprofissional no contexto brasileiro: Algumas reflexões. Interface (Botucatu). 2016;20(56):197-8.

3. Peduzzi M. O SUS é interprofissional. Interface (Botucatu). 2016;20(56):199-201.

4. Batista NA. Educação Interprofissional em Saúde : Concepções e Práticas. Cad FNEPAS. 2012;2:25-8.

5. Peduzzi M, Norman IJ, Germani ACCG, Silva JAM, Souza GC. Educação interprofissional: formação de profissionais de saúde para o trabalho em equipe com foco nos usuários. Rev Esc Enferm USP. 2013;47(4):977-83.

6. Buring SM, Bhushan A, Broeseker A, Conway S, Duncan-hewitt W, Hansen L, et al. Interprofessional Education: definitions, student competencies, and guidelines for 
implementation. Am J Pharm Educ. 2009;73(4):1-8.

7. Reeves S, Perrier L, Goldman J, Freeth D, Zwarenstein M. Interprofessional education: effects on professional practice and healthcare outcomes (update). Cochrane Database Syst Rev [Internet]. 2013;3.47p. [Acesso em 10/10/ 2018]. Disponível em: http://www.ncbi. nlm.nih.gov/pubmed/23543515\%5Cnhttp://on linelibrary.wiley.com/doi/10.1002/14651858. CD002213.pub2/pdf/standard\%5Cnhttp://ww w.ncbi.nlm.nih.gov/pubmed/23543515

8. Costa T, Lopes S, Fernández-Limós F, Amante MJ, Lopes PF. A Bibliometria e a Avaliação da Produção Científica: indicadores e ferramentas. ACTAS - Congr Nac Bibl Arq e Doc 11. 2012;(11):1-7.

9. Oliveira CM, Batista NA, Batista SHSS, Uchôa-Figueiredo LR. A escrita de narrativas e o desenvolvimento de práticas colaborativas para o trabalho em equipe. Interface (Botucatu). 2016;20(59):1005-14.

10. Perego MG, Batista NA. Aprendizagens compartilhadas na Residência Multiprofissional em Saúde. Tempus. 2016; 10(4):39-51.

11. Souto S, Batista SH. A educação interprofissional na formação em psicologia: olhares de estudantes. Psicol Ciência e Profissão. 2014;34(1):32-45.

12. Casanova IA, Batista NA, Ruiz-Moreno L. Formação para o trabalho em equipe na residência multiprofissional em saúde. ABCS Heal Sci. 2015;40(3):229-33.

13. Santos GM, Batista SHSS. Monitoria acadêmica na formação em/para a saúde: desafios e possibilidades no âmbito de um currículo interprofissional em saúde. ABCS Heal Sci. 2015;40(3):203-7.

14. Costa MV, Patrício KP, Câmara AMCS, Azevedo GD, Batista SHS da S. Pró-Saúde e PET-Saúde como espaços de educação interprofissional. Interface (Botucatu). 2015;19(Supl. 1):709-20.

15. Ellery AEL. Interprofissionalidade na
Estratégia Saúde da Família: condições de possibilidade para a integração de saberes e a colaboração interprofissional. 2012.

16. Ellery AEL, Pontes JRS, Loiola FA. Campo comum de atuação dos profissionais da Estratégia Saúde da Família no Brasil: um cenário em construção. Physis. 2013;23(2):415-37.

17. Barros ER, Ellery AEL. Inter-professional collaboration in an Intensive Care Unit: challenges and opportunities. Rev Rene. 2016;17(1):10-9.

18. Germani ACCG, Oliveira AA, Ferreira HP, Yano AC, Sancho GM. The use of information technology in the postgraduate course in the health. Rev Med (São Paulo). 2013;92(2):97103.

19. Germani ACCG, Oliveira AAP, Chiesa AM, Ferreira Júnior M. Avaliação de tecnologias educacionais digitais para a formação de promotores de saúde em uma experiência interprofissional. J Bras Tele. 2014;3(1):20010.

20. Souza GC, Peduzzi M, Silva JAM, Carvalho BG. Trabalho em equipe de enfermagem: circunscrito à profissão ou colaboração interprofissional? Rev Esc Enferm USP. 2016;50(4):642-9.

21. Lima FS, Amestoy SC, Jacondino MB, Trindade LL, Silva CN, Fuculo Junior PRB. Exercício da liderança do enfermeiro na estratégia saúde da família. J Res Fundam Care Online. 2016;8(1):3893-906.

22. Fernandes HN, Thofehrn MB, Porto AR, Amestoy SC, Jacondino MB, Soares MR. Relacionamento interpessoal no trabalho da equipe multiprofissional de uma unidade de saúde da família. J Res Fundam Care Online. 2015;7(1):1915-26.

23. Nuto SAS, Lima Júnior FCM, Camara AMCS, Gonçalves CBC. Avaliação da disponibilidade para aprendizagem interprofissional de estudantes de Ciências da Saúde. Rev Bras Educ Med. 2017;41(1):50-7.

24. Forte FDS, Morais HGF, Rodrigues SAG, 
Santos JS, Oliveira PFA, Morais MST, et al. Educação interprofissional e o programa de educação pelo trabalho para a saúde/Rede Cegonha: potencializando mudanças na formação acadêmica. Interface (Botucatu). 2016;20(58):787-96.

25. Araújo TAM, Vasconcelos ACCP, Pessoa TRRF, Forte FDS. Multiprofissionalidade e interprofissionalidade em uma residência hospitalar: o olhar de residentes e preceptores. Interface (Botucatu). 2017;21(62):601-13.

26. Câmara AMCS, Cyrino AP, Cyrino EGC, Azevedo GD, Costa MV, Bellini MIB, et al. Interprofessional education in Brazil: Building synergic networks of educational and healthcare processes. Interface (Botucatu) [Internet]. 2016;30(2):135-7.

27. Reeves S. Por que precisamos da educação interprofissional para um cuidado efetivo e seguro. Interface (Botucatu). 2016;20 (56):185-97.

28. Batista NA, Batista SHSDS. Educação interprofissional na formação em Saúde: Tecendo redes de práticas e saberes. Interface Commun Heal Educ. 2016;20(56):204-6.

29. Reeves S. Reply. Interface - Comun Saúde, Educ [Internet]. 2016;20(56):205-6. [Acesso em: 10 out. 2018]. Disponível em: http://www .scielo.br/scielo.php?script=sci_arttext\&pid=S 1414-32832016000100205\&lng=pt\&nrm= iso\&tlng=en

30. Costa MV, Vilar MJ, Azevedo GD, Reeves S. Interprofessional education as an approach for reforming health professions education in Brazil: Emerging findings Interprofessional education as an approach for reforming health professions education in Brazil: emerging findings. J Interprof Care. 2014;28(4):379-80.

31. Sidone G, Haddad EA, Mena-chalco JP. A ciência nas regiões brasileiras: evolução da produção e das redes de colaboração científica Science in Brazilian regions: evelopment of scholarly production and research. TransInformação. 2016;28(1):15-31.

32. Bosi MLM. Pesquisa qualitativa em saúde coletiva: panorama e desafios. Cien Saude Colet. 2012;17(3):575-86.

33. Minayo MCDS. Análise qualitativa: teoria, passos e fidedignidade. Cien Saude Colet. 2012;17(3):621-6.

34. Pope C, Mays N. Métodos qualitativos na pesquisa em saúde. In: Pesquisa qualitativa na atenção à saúde. 3a ed. Porto Alegre: Artmed; 2009. p. 11-21.

35. Frenk J, Chen L, Bhutta ZA, Cohen J, Crisp N, Evans T, et al. Health professionals for a new century: Ttransforming education to strengthen health systems in an interdependent world. Lancet. 2010;376(9756):1923-58.

36. Barr H. Competent to collaborate: Towards a comptence-based model for interprofessional education. J Interprof Care. 1998;12(2):181-8.

37. Barros AJD. Produção científica em saúde coletiva: perfil dos periódicos e avaliação pela Capes. Rev Saude Publica. 2006;40:43-9.

38. Elias FS, Souza L. Indicadores para monitoramento de pesquisa em saúde no Brasil. Ciência da Informação. 2006;35:218-26.

39. Batista NA, Batista SH, Rossit RAS, Gerab IF da S. A educação interprofissional na graduação em saúde: preparando profissionais para o trabalho em equipe e para a integralidade no cuidado. Atas do IX Encontro Nac Pesqui em Educ em Ciências - IX ENPEC. 2013;1-6.

40. Reeves S, Pelone F, Harrison R, Goldman J, Zwarenstein M. Interprofessional collaboration to improve professional practice and healthcare outcomes (Review). Cochrane Libr. 2017;(6):1-48.

41. Reeves S, Fletcher S, Barr H, Birch I, Boet S, Davies N, et al. A BEME systematic review of the effects of interprofessional education: BEME Guide No. 39. Med Teach. 2016;(39):1-27.

\section{Correspondência para:}

Ramona Fernanda Ceriotti Toassi

e-mail: ramona.fernanda@ufrgs.br

Rua Ramiro Barcelos, 2492 - 3o andar

90035-003 Porto Alegre/RS 